\title{
Autismo: Revisión Conceptual
}

\author{
Autism: Conceptual review
}

\section{Anaid García-Franco ${ }^{a}$, Oliva Alejandra Alpizar-Lorenzo ${ }^{b}$, Gelacio Guzmán-Diaz ${ }^{c}$}

\begin{abstract}
:
An accomplishment about of Autism Spectrum Disorder (ASD), based on a revision of the Diagnostic and Statistical Manual of Mental Disorders (DSM V) and in articles of scientific magazines like the Latinamerican Magazine of Psychology, for explain the incorporation of the word "Spectrum" to concept of ASD. With the incorporation of this variant at the concept of ASD, it tipology is too modificated, including all the types of autism previously known in a unique concept: the ASD.
\end{abstract}

Keywords:

Autism Spectrum Dosorder, Conceptualization, Typology, Simptomatology, Differential diagnosis

Resumen:

Se realiza una conceptualización teórica del Trastorno del Espectro Autista (TEA), basada en la revisión del Manual Diagnóstico y Estadístico de los Trastornos Mentales (DSM V) y en artículos de revistas científicas como la Revista Latinoamericana de Psicología, donde se explica la inclusión de la palabra "espectro" al concepto del TEA. Con la inclusión de esta variante al concepto del TEA, se modifica también su tipología, englobando a todos los tipos de autismo antes conocidos en uno solo: el TEA.

\section{Palabras Clave:}

Trastorno del espectro autista, conceptualización, tipología, sintomatología, Diagnóstico diferencial.

\section{INTRODUCCIÓN}

El autismo, al igual que la mayoría de los trastornos psicológicos, ha sido estudiado desde años atrás por diversos psicólogos, neurólogos, psiquiatras y profesionales de la salud; a lo largo de estos estudios se ha intentado establecer un concepto real y homogéneo que describa acertadamente el trastorno y sus características; sin embargo, los conceptos construidos desde épocas de antaño y hasta la actualidad siguen siendo difusos y diferentes entre sí, recalcando en cada uno de ellos aspectos diferentes que cada autor considera relevantes para la definición del trastorno autista.

El término autismo es utilizado por primera vez en 1911 por el psiquiatra Suizo Eugen Bleuler (1857-1939) en su monografía Dementia praecox oder Gruppe der Schizophrenien redactada para el Tratado de Psiquiatría y publicada en Viena (1).

\begin{abstract}
Este término, creado por Bleuler, tiene una etimología griega "autos" que significa "sí mismo" "por cuenta propia", e "ismo", un sufijo que denota acción o estado. Para Bleuler, el autismo está caracterizado por el repliegue de la vida mental del sujeto sobre sí mismo, llegándose a la constitución de un mundo cerrado separado de la realidad exterior y a la dificultad extrema o la imposibilidad de comunicarse con los demás que de allí resulta (1).

Como se mencionó anteriormente, no existe una definición homogénea y específica sobre el autismo, no obstante, López, Rivas y Toboaba (2) lo definen, en términos generales, como: "un trastorno neuropsicológico [...] que se manifiesta con una alteración cualitativa de la interacción social y de la comunicación así como con unos patrones comportamentales restringidos, repetitivos y estereotipados con distintos niveles de gravedad (p.557)".
\end{abstract}

Así mismo, resalta también dentro de éstas definiciones,

\footnotetext{
Autor de Correspondencia, Universidad Autónoma del Estado de Hidalgo, Escuela Superior de Atotonilco de Tula, Email: ga320465@uaeh.edu.mx

b Universidad Autónoma del Estado de Hidalgo, Escuela Superior de Atotonilco de Tula, ,Email:al314672@uaeh.edu.mx

c Universidad Autónoma del Estado de Hidalgo, Escuela Superior de Atotonilco de Tula, ORCID: 0000-0003-4869-8658, Email: 
Leo Kanner (1894-1981) quien diferencia el autismo de los trastornos esquizofrénicos y lo define como una alteración innata del contacto afectivo (3).

Actualmente, el trastorno autista ha adquirido una nueva concepción, dándose a conocer como Trastorno del Espectro Autista (TEA). Éste término fue propuesto por la psiquiatra británica Lorna Wing (1928-2014) quien refiere que el autismo es un trastorno de espectro, es decir, que involucra una extensa variedad de síntomas, habilidades y niveles de discapacidad que puede tener el individuo que los padece. Derivado de ésta aportación, la American Psychological Association (APA) incluye en la quinta edición del Manual Diagnóstico y Estadístico de los Trastornos Mentales (DSM-V) al autismo en la clasificación de los trastornos del neurodesarrollo, con el nombre de Trastornos del Espectro Autista (4)

\section{Tipología}

Al igual que ocurre con la definición del autismo, la clasificación y tipología del mismo trastorno han pasado por una evolución significativa que ha causado gran polémica y confusión entre la comunidad de psicólogos, psiquiatras y neurólogos.

En la cuarta edición del DSM, el TEA se encontraba dentro de la clasificación de los Trastornos Degenerativos del Desarrollo (TGD), los cuales incluían el Trastorno Autista, Trastorno de Rett, Trastorno Desintegrativo Infantil, Trastorno de Asperger y el Trastorno Generalizado del Desarrollo No Especificado (5). Sin embargo y en consecuencia de los resultados de las revisiones y actualizaciones de la APA en el DSM V(4), en ésta última edición se modifican los diferentes subtipos de TGD. El trastorno autista, el síndrome de Asperger y el trastorno generalizado del desarrollo no especificado se fusionan conformando el Trastorno del Espectro Autista. La clasificación TGD también es modificada, y se sustituye por lo que ahora aparece como Trastornos del neurodesarrollo. El Síndrome de Rett es modificado dentro de la clasificación debido a que tiene una base genética y por tal razón deja de considerarse una enfermedad mental; mientras que el Trastorno Desintegrativo Infantil no ha sido validado del todo para incluirse en la tipología del TEA.

\section{Etiopatogenia}

A pesar de las investigaciones sobre la etiología del autismo que se han realizado hasta el momento, no se ha podido detectar una causa específica que lo origine, en realidad, las conclusiones etiopatogénicas a las que han llegado los investigadores son ambiguas, difusas e inespecíficas.

De acuerdo con Varela, Ruiz, Vela, Munive y Hernández (6) para explicar las causas que hasta ahora han sido descubiertas, se ha dividido al TEA en dos subgrupos; el autismo primario o idiopático y el autismo secundario o sindromático. El autismo primario tiene una base genética inespecífica, heredada por los padres, es decir, la participación simultánea de diferentes genes. La participación de factores genéticos en el autismo primario es considerablemente importante. A diferencia del autismo primario, en el secundario se observa en afecciones neurológicas, que en muchos casos tienen una base genética. Se considera secundario cuando se identifica una entidad patológica como su causa. Las causas del autismo secundario o sindromático son: trastornos genéticos, trastornos congénitos del metabolismo, infecciones congénitas o adquiridas, encefalopatía hipóxico isquémica, y displasias corticales, entre otras.

Otra clasificación del TEA es la propuesta por López, Rivas y Toboada (2) la cual propone que las teorías etiológicas del autismo se dividen en dos grupos; el primero se centra en las causas patogénicas y hace referencia a anomalías en el entorno psicológico o a problemas orgánicos relacionados con anomalías genéticas. El segundo grupo identifica los desórdenes psicológicos y fisiológicos como rasgos etiológicos, dentro de los cuales destaca la organización cerebral diferente, los trastornos neurofisiológicos y los déficits cognitivos, perceptivos y sensoriomotores.

La concepción psicogénica de la etiología del autismo surgió antes que la concepción biológica en el ámbito de la investigación, sin embargo y de acuerdo con estudios más recientes, la propuesta de Varela y Cols.(6) acerca del autismo idiopático y el sindromático es más aceptada en el grupo de los estudiosos del tema en comparación con la psicogénica que retoman Lopéz y Cols. (2), debido a que tiene un sustento científico y biológico, mientras que la psicogénica tiene un sentido más subjetivo, por lo tanto podría ser desvalorizada y rechazada.

Vargas y Navas (7) proponen tres clasificaciones etiopatogénicas del TEA; la primera es la etiología genética, en la cual explica que existen al menos diez genes que se multiplican e interactúan entre sí provocando una variación fenotípica en el genoma humano. Señala también que existe una relación causal entre síndromes neurogenéticos, como el síndrome del $X$ frágil o el síndrome de Rett y el autismo.

La segunda clasificación que Vargas y Navas(7) mencionan es la neurobiológica, la cual propone que el origen del TEA puede deberse a alteraciones en el desarrollo cerebral que pueden originarse durante el periodo prenatal y perduran toda la vida. De manera similar, las alteraciones en el cerebelo y específicamente en el número y tamaño de las células de Purkinje sugieren un trastorno evolutivo en las conexiones sinápticas, lo que supone un déficit mental.

La tercer clasificación etiopatogénica propuesta por Vargas y Navas (7) tiene su base en la teoría psicógena, la cual explica la génesis del TEA desde una postura psicoanalítica, partiendo de la idea de un "autismo inducido", producto de las primeras relaciones madrehijo. Este enfoque supone que el niño al nacer es completamente regular, y que los síntomas del TEA se desarrollan a partir de pautas de crianza defectuosas por parte de los padres. Sin embargo y afortunadamente, este postulado carece de bases científicas y empíricas comprobables.

Reynoso, Rangel y Melgar (8) consideran que el TEA tiene un componente genético, asimismo, mencionan al TEA como una enfermedad poligénica y multifactorial en la que las variaciones genéticas al interactuar con los 
factores ambientales dan como resultado un fenotipo específico; el cual podría ser el causante del TEA.

\section{Sintomatología}

EI TEA presenta diversos síntomas a lo largo de la vida del sujeto que lo posee, sin embargo, entre los más significativos, de acuerdo con el DSM IV (5) se encuentran: 1) la presencia de un desarrollo marcadamente anormal o deficiente de la interacción y comunicación sociales y 2) un repertorio sumamente restrictivo de actividades e intereses. Estas manifestaciones del trastorno varían de acuerdo a la edad cronológica y al nivel de desarrollo del sujeto, así como del sexo. Por ejemplo, un niño puede mostrar inicialmente especial aversión a los estímulos sociales, y durante su desarrollo ir adquiriendo habilidades sociales que le permitan una mejor disposición a participar pasivamente en la interacción social, lo cual culminaría en un adulto sociable que tuvo como inicio una infancia asocial. Los síntomas en ambas etapas se desarrollan de manera distinta. En cuanto al sexo, diversas investigaciones demuestran que los varones tienen una mayor predisposición a presentar un diagnóstico de TEA.

En las actualizaciones que la APA ha realizado al DSM, se ha modificado la sintomatología del TEA, para describir sucintamente la sintomatología actual. Para describir la sintomatología el DSM V (4) utiliza especificadores de gravedad que varían de acuerdo al contexto y fluctúan con el tiempo. Por consiguiente los especificadores que se toman en cuenta son tres: "con o sin deterioro intelectual asociado", "con o sin deterioro del lenguaje que lo acompaña" y "asociado con una condición médica o genética conocida o factor ambiental”. El primer especificador se refiere a la comprensión del perfil intelectual de una persona con TEA para la interpretación de las características de diagnóstico. El segundo implica la evaluación y descripción del nivel actual de funcionamiento verbal de la persona con TEA, y por último, el tercer especificador se utiliza cuando el individuo tiene un trastorno genético conocido, un trastorno médico o un historial de exposición ambiental. La sintomatología es descrita por diversos autores de manera diferente, sin embargo, existen síntomas característicos que deben ser tomados en cuenta al hablar de sintomatología del TEA. Por ejemplo, López y cols. (2) mencionan que los sujetos con TEA muestran tres características principales conocidas como "la triada del autismo". La primera corresponde a deficiencias de la interacción social marcadas por una notable afectación de la práctica de comportamientos no verbales. La segunda característica indica que existe una notable y persistente alteración de la comunicación, que afecta a las habilidades verbales y no verbales, lo cual causa dificultad en la adquisición del lenguaje o su ausencia total. Y por último, la tercera característica menciona que los individuos con TEA presentan patrones de comportamiento, intereses y actividades restringidas, repetitivas y estereotipadas, y se adhieren de manera inflexible a sus rituales o rutinas específicas, tanto que intentar modificarlos puede resultarles sumamente conflictivo e inaceptable.
Aunque los síntomas no son descritos exactamente igual en cada caso, estos reflejan un mismo sentido; es decir, un individuo diagnosticado con TEA va a presentar conductas estereotipadas, dificultades en la interacción y comunicación social $y$, dependiendo del nivel de funcionalidad de TEA puede o no presentar deficiencia intelectual. Existen algunos factores relacionados con los síntomas, como la edad cronológica, el nivel de desarrollo y el sexo, de los cuales se considera más significativo el nivel de desarrollo, dado que durante el desarrollo, al sujeto se le pueden implantar conductas que le ayuden a ser más funcional $y$ a disminuir las conductas disfuncionales del TEA, y por tanto, los síntomas y de esta manera mejorar la calidad de vida del sujeto.

La sintomatología del TEA incluye varios síntomas, algunos de ellos no son exclusivos del TEA, es decir, pueden presentarse en otros tipos de trastornos y por ende, causar cierta confusión; debido a esto, es necesario realizar un diagnóstico específico del TEA y asegurarse de que el cuadro clínico se cumple. A continuación se presentan los puntos que se deben considerar al realizar un diagnóstico del TEA.

\section{Diagnóstico}

Desde la creación de la primera edición del DSM hasta la versión actual se han realizado diversas modificaciones en las conceptualizaciones, criterios diagnósticos, sintomatología, etiología, características, comorbilidad y prevalencia de los distintos trastornos incluidos en el manual; el TEA no es una excepción, ha pasado por un proceso exhaustivo de actualización en varios ámbitos que establece el DSM y tal proceso ha causado controversias significativas.

Para realizar las actualizaciones de los criterios diagnósticos del TEA en el DSM fue necesario tomar en cuenta las aportaciones de asociaciones como la APA y en segundo lugar por la Asociación Española de Profesionales del Autismo (AETAPI). La APA es una de las encargadas de las modificaciones y actualizaciones que se realizan en cada edición del DSM. La AETAPI no interviene en tal proceso de actualización y modificación, sin embargo, se dedica a emitir valoraciones $\mathrm{y} / \mathrm{u}$ opiniones acerca de la factibilidad que tienen tales actualizaciones para los individuos diagnosticados con TEA. En el caso de la última actualización del DSM, La AETAPI valora positivamente que se haya asumido una visión dimensional del trastorno, refiriéndose a él como un "espectro", así como la unificación de las alteraciones sociales y comunicativas en una misma dimensión y la especificación más concreta de los criterios diagnósticos(9).

Sin embargo, la AETAPI valora negativamente algunos elementos que no parecen claros en el DSM-V, como el juego simbólico -que se ha eliminado de los criterios diagnósticos-, y las alteraciones emocionales que tienen muy poca presencia en los criterios diagnósticos. También valora negativamente la excesiva especificidad del dominio de alteración referido a la presencia de patrones de conductas, intereses y actividades restringidas y repetitivas, ya que al exigir que una persona deba presentar alteraciones en dos de las cuatro áreas de la alteración, pues hace muy probable que muchas 
personas no reciban el diagnóstico de TEA por presentar alteración en una sola área, mientras que con el DSM-IV sí se diagnostica (9).

De acuerdo con Aguiar, Mainegra, García \& Hernández (10) para establecer un diagnóstico de TEA de acuerdo con los criterios del DSM-V, es indispensable realizar una evaluación integral del sujeto, la cual debe incluir los distintos contextos donde se desenvuelve, como el contexto familiar, escolar y el clínico. EI DSM-V plantea que el diagnóstico de TEA se establece de acuerdo al nivel de adaptación del sujeto en los contextos mencionados anteriormente, y debe cumplir los siguientes criterios:

A. Déficit persistentes en la comunicación y en la interacción social en diversos contextos, no atribuibles a un retraso general del desarrollo, manifestando simultáneamente los tres déficit siguientes:

1. Déficit en la reciprocidad social y emocional; pasando por la reducción de intereses, emociones y afectos compartidos, hasta la ausencia total de iniciativa en la interacción social.

2. Déficit en las conductas de comunicación no verbal que se usan en la comunicación social; pasando por anormalidades en el contacto visual y el lenguaje corporal, o déficit en la comprensión y uso de la comunicación no verbal, hasta la falta total de expresiones o gestos faciales.

3. Déficit en el desarrollo y mantenimiento de relaciones adecuadas al nivel de desarrollo; que pueden abarcar desde dificultades para mantener un comportamiento apropiado a los diferentes contextos sociales, hasta la aparente ausencia de interés en las otras personas.

B. Patrones de comportamiento, intereses o actividades restringidas y repetitivas que se manifiestan al menos en dos de los siguientes puntos:

1. Habla, movimientos o manipulación de objetos estereotipada o repetitiva

2. Excesiva fijación con las rutinas, los patrones ritualizados de conducta verbal y no verbal, o excesiva resistencia al cambio

3. Intereses altamente restrictivos y fijos de intensidad desmesurada

4. Hiper o hipo reactividad a los estímulos sensoriales o inusual interés en aspectos sensoriales del entorno

C. Los síntomas deben estar presentes en la primera infancia (pero pueden no llegar a manifestarse plenamente hasta que las demandas sociales exceden las limitadas capacidades).

D. La conjunción de síntomas limita el funcionamiento cotidiano del sujeto.

\section{Diagnóstico diferencial}

Una vez definidos los criterios para diagnosticar el TEA es necesario establecer un diagnóstico diferencial. El diagnóstico diferencial se realiza con la finalidad de realizar un diagnóstico correcto, ya que en muchas ocasiones podrían confundirse los criterios clínicos. El DSM-V (año) establece los siguientes diagnósticos diferenciales del TEA:

Síndrome de Rett. Rodríguez, Delgado \& González (11) se refieren al síndrome de Rett como un trastorno del neurodesarrollo caracterizado por regresión psicomotora, comportamiento autista, desaceleración del crecimiento de la cabeza (microcefalia postnatal), convulsiones, pérdida de las funciones propositivas manuales y movimientos repetitivos estereotipados de las manos; y toman en cuenta una característica destacada en la incidencia del síndrome, aseverando que es más común en el sexo femenino; las causas exactas de tal fenómeno aún son desconocidas.

El síndrome de Rett debe su nombre a debe su nombre a su descubridor: el austriaco Andreas Rett; este síndrome se encontraba dentro de la clasificación de los Trastornos Generalizados del Desarrollo del DSM-IV; en donde se establecía que las principales características de este grupo de trastornos son la presencia de un deterioro severo en las áreas de habilidades sociales, habilidades para la comunicación y la presencia de conductas, intereses o actividades estereotipadas(12); sin embargo, la nueva edición del DSM ya no lo incluye como trastorno y lo clasifica como síndrome.

Por lo tanto, para diferenciar un diagnóstico de TEA de un síndrome de Rett, la característica principal a tomar en cuenta es la regresión; es decir, mientras que en el TEA el niño nace con las características propias establecidas en el cuadro clínico, en el síndrome de Rett el niño tiene inicialmente un desarrollo normal, el cual se ve afectado por la mutación del gen MECP2 provocando una regresión psicomotora (11)

Mutismo selectivo. Lahoza (13) se refiere al mutismo selectivo como la dificultad que presentan algunos niños para comunicarse verbalmente en entornos y situaciones poco familiares $y / 0$ con personas poco conocidas, sin embargo, los niños tienen una competencia lingüística y comunicativa adecuada para su edad, la cual se manifiesta habitualmente en el entorno familiar pero no en otros ambientes poco conocidos. Muchos de los niños con mutismo selectivo suelen presentar además algunos rasgos de personalidad característicos, como timidez, retraimiento social, perfeccionismo o dependencia. EI mutismo selectivo es un trastorno de la conducta que se inicia en la infancia y se caracteriza por la dificultad del niño para interactuar verbalmente con determinadas personas y en determinadas situaciones.

Rodríguez y Saval (14)clasifican al mutismo selectivo dentro de los trastornos de ansiedad social del DSM-V y mencionan que se caracteriza por la inhibición selectiva del habla en situaciones en las que existe expectativa de hablar y que interfiere en los logros educativos o laborales o en la comunicación social.

Cabe recalcar que ambas clasificaciones consideran al mutismo selectivo en dos áreas distintas, la primera, en un área conductual, es decir, como un trastorno de la conducta iniciado durante la infancia, mientras que la segunda indica al mutismo selectivo en el área de los trastornos de ansiedad social. Sin embargo, se considera con un mayor alcance a la clasificación definida dentro del DSM-V, es decir, clasificar al mutismo selectivo dentro de los trastornos de la ansiedad social. De la misma manera, es una clasificación que se considera más "acertada" debido a que el mutismo selectivo no implica simplemente una conducta, sino que implica el área del lenguaje, y el lenguaje a su vez, es una habilidad social. Tomando en cuenta lo anterior, se puede establecer la diferenciación del diagnóstico del TEA. Un diagnóstico de 
TEA se diferencia del mutismo selectivo ya que el mutismo selectivo no presenta patrones de comportamiento; es decir, intereses 0 actividades restringidas y repetitivas, déficit en la reciprocidad social y emocional o déficit de atención; como tal, el mutismo selectivo está más relacionado a una anomalía en la comunicación.

Trastornos del lenguaje y trastorno de comunicación social. El niño afectado generalmente exhibe habilidades de comunicación apropiadas en ciertos contextos y configuraciones, incluso en entornos donde el niño está mudo, la reciprocidad social no se altera, tampoco hay patrones de conducta restringidos o repetitivos.

En algunas formas del trastorno del lenguaje, puede haber problemas de comunicación y dificultades sociales. Sin embargo, el trastorno del lenguaje específico generalmente no se asocia con anomalías comunicación no verbal, o con la presencia de patrones restringidos y repetitivos de comportamiento, intereses o actividades (4).

\section{Discapacidad intelectual sin trastorno del espectro autista.}

La definición de discapacidad intelectual, al igual que la definición del TEA, ha pasado por un proceso de actualización, en el que se han considerado diversos factores para poder establecer la definición que actualmente conocemos.

Luckasson, Borthwick-Duffy, Buntinx, Coulter, Craig, Reeve, Schalock, Snel, Spitalnik, Spreat, y Tasse (15)actualizan el concepto de discapacidad intelectual (DI) estableciendo que: "el retraso mental es una discapacidad caracterizada por limitaciones significativas en el funcionamiento intelectual y la conducta adaptativa tal como se ha manifestado en habilidades prácticas, sociales y conceptuales. Esta capacidad comienza antes de los 18 años (p. 327)".

De acuerdo con la APA, en el DSM-V (4) se establece que:

"La discapacidad intelectual (trastorno del desarrollo intelectual) es un trastorno que comienza durante el período de desarrollo y que incluye limitaciones del funcionamiento intelectual como también del comportamiento adaptativo en los dominios conceptual, social y práctico (p. 17)".

La primer diferencia que nos encontramos al establecer un diagnóstico de TEA y no uno de DI es el déficit en la interacción y la comunicación social; un niño diagnosticado con TEA presentará dificultades en la reciprocidad social y emocional, en la comunicación no verbal, y en el mantenimiento de las relaciones sociales; mientras que en un niño con DI, éstas condiciones no difieren de las normales. De igual manera, los patrones de comportamiento, intereses y actividades restringidas o repetitivas que presenta el TEA no se presentan en un diagnóstico de DI; por lo cual, si el niño que será diagnosticado no presenta tales síntomas y sus deficiencias se encuentran situadas en las funciones intelectuales, como el razonamiento, la resolución de problemas, la planificación, el pensamiento abstracto, el juicio, el aprendizaje académico y el aprendizaje a partir de la experiencia, entonces el diagnóstico que será establecido será el de DI.

\section{Trastorno estereotípico del movimiento}

Fernández (16) define a la estereotipia como "una actividad motora organizada, repetitiva, no propositiva, que se lleva a cabo exactamente de la misma forma en cada repetición (Pp. 54)"y señala que el DSM-IV la define como "conducta motriz que es repetitiva, a menudo aparentemente orientada, y no funcional". La definición sugerida por Fernández (16) así como la establecida en el DSM-IV no consideran la ritmicidad como una condición necesaria, lo cual ayuda a diferenciarla del síndrome de Tourette, por ejemplo. Las estereotipias pueden ser rítmicas o no rítmicas y pueden clasificarse, según el tiempo de permanencia, como transitorias o persistentes. Las estereotipias persistentes son frecuentes en sujetos patológicos, es decir, sujetos con defectos sensoriales, malformaciones cerebelosas, autismo, disturbios emocionales, deprivación ambiental grave; y en lactantes institucionalizados. Se realizan habitualmente cuando el niño está en un estado de excitación o de tensión o cuando el niño se aburre o se concentra mentalmente. Las estereotipias más usuales en estos niños son aletear las manos, juguetear con sus manos, entrecruzar los dedos, pellizcarse la piel, introducir repetidamente sus dedos en orificios corporales o golpearse partes de su propio cuerpo.

Las estereotipias se encuentran entre las características diagnósticas del trastorno del espectro autista, por lo que un diagnóstico adicional de movimiento estereotípico no ocurre cuando tales comportamientos repetitivos se explican mejor por la presencia del trastorno del espectro autista. Sin embargo, cuando las estereotipias causan autolesiones y se convierte en el enfoque de tratamiento, ambos diagnósticos pueden ser apropiados.

Trastorno hiperactivo y déficit de atención. Se refiere a las anormalidades de la atención (demasiado enfocadas o fácil de distraer), son comunes en las personas con trastorno del espectro autista, como lo es la hiperactividad. Sin embargo, un diagnóstico de trastorno por déficit de atención / hiperactividad (TDAH) debe ser considerado cuando las dificultades atencionales o la hiperactividad exceden a las que se ven típicamente en individuos de edad mental comparable(4).

Esquizofrenia. La esquizofrenia con inicio en la infancia, generalmente se desarrolla después de un período de desarrollo normal o casi normal. Se ha descrito un estado prodrómico en el cual el deterioro de la capacidad e intereses y creencias atípicos, que podrían confundirse con déficits sociales vistos en el trastorno del espectro autista. Alucinaciones y delirios, que definen las características de la esquizofrenia no son características del trastorno del espectro autista, por lo tanto, se deben tener muy claros los criterios clínicos (4).

\section{Conclusiones}

La conceptualización del Autismo ha evolucionado a lo largo de los años, desde el concepto que estableció Bleuler, basándose en un enfoque psiquiátrico, en donde caracteriza al autismo como un repliegue de la vida mental del sujeto que lo coloca en condiciones de aislamiento, hasta la conceptualización que da a conocer la Asociación Americana de Psiquiatría (APA) en la quinta Edición del Manual Diagnóstico y Estadístico de los Trastornos Mentales (DSM V) en donde incluye el concepto de "espectro" para englobar los tipos de autismo 
que se consideraban en el DSM IV homologando sus síntomas y cuadros clínicos en uno sólo. La actualización del concepto del TEA tiene una importancia significativa en el área de la psicología, la psiquiatría, debido a que, se debe diagnosticar de forma correcta a los sujetos que son el objeto de estudio del psicólogo y del psiquiatra, para establecer un diagnóstico correcto del trastorno, es necesario conocer los nuevos criterios que establece el DSM-V tanto en la conceptualización como en su sintomatología y diagnóstico.

Sin embargo, el diagnóstico no es el único apartado en el que recae la conceptualización del TEA; para los psicólogos y profesionales que trabajan en el campo de la psicología educativa, el conocer la conceptualización, sintomatología y criterios diagnósticos del TEA de manera adecuada les ayudará a reconocer las conductas de los infantes e identificar si tales conductas forman parte de los criterios diagnósticos del TEA y de esta forma poder entender el por qué el infante probablemente no está teniendo un desarrollo adecuado en los diversos ámbitos en que se incluye. A través de la identificación de los síntomas y la elaboración del diagnóstico, el psicólogo podrá brindar una mejor y más adecuada atención al infante, de manera que estará colaborando con su desarrollo y le propiciará una mejor calidad de vida.

Es necesario también, prestar especial atención al diagnóstico diferencial del TEA, ya que, como se vio a lo largo del artículo, existen varios trastornos y síndromes con los que el TEA podría ser confundido. Sin embargo, los criterios clínicos presentados anteriormente deben ser revisados por un profesional siempre con cautela y atención para no generar un diagnóstico erróneo. De no ser así, el sujeto diagnosticado podría sufrir afectaciones cognitivas o conductuales durante su tratamiento.

\section{REFERENCIAS}

1. Garrabé de Lara J. El autismo: Historia y clasificaciones. Salud mental. junio de 2012;35(3):257-61

2. Eva María Taboada Ares SLG. Revisiones sobre el autismo. Revista Latinoamericana en Psicología. 2009;41:555-70.

3. Artigas-Pallares J, Paula I. El autismo 70 años después de Leo Kanner y Hans Asperger. Revista de la Asociación Española de Neuropsiquiatría. septiembre de 2012;32(115):567-87.

4. American Psychiatric Association. DIAGNOSTIC AND STATISTICAL MANUAL OF MENTAL DISORDERS. 5. ${ }^{\mathrm{a}}$ ed. 2013.

5. American Psychiatric Association. Manual diagnóstico y estadístico de los trastornos mentales. IV. España; 1995. 894 p.

6. Antunes DMVaGB gloria H, Matilde Ruiz García, Amieva MV, Leticia Munive Baez. Conceptos actuales sobre la etiología del autismo. Acta Pediátrica de México. 2011;213-22.

7. María Jesús Vargas Balderas, Wendy Navas Orozco. AUTISMO INFANTIL. Revista cúpula. 2012;44-58.

8. César Reynoso, María José Rangel, Virgilio Melgar. El Trastorno de Espectro autista: Aspectos etiológicos, diagnósticos y terapeúticos. Revista Médica del Instituto Mexicano del Seguro Social. 2017;214-22.
9. Patricia García Tabuenca. Trastorno del espectro autista (TEA). Anuario del Centro de la Universidad Nacional de Educación a Distancia en Calatayud. 2016;(22):149-62.

10. Giselvis Aguiar Aguiar Yenira Hernández Fonticiella, Fernández DM, Olivia García Reyes. Diagnóstico en niños con trastornos del espectro autista en su desarrollo en la comprensión textual. Revista de Cioencias Médicas de Pinar de Río. 2016;20(6):72937.

11. Fernanda Rodríguez Ramos, Wilmer Delgado Luengo, Sandra González Ferrer. ASPECTOS CLÍNICOS DEL SÍNDROME DE RETT EN PACIENTES VENEZOLANOS. Saber, Universidad de Oriente, Venezuela. 2016;28(4):726-35.

12. Jorge J. Gómez Gude, Maximina Rodríguez Fernández, Emma Blanco. DESCRIPCIÓN DEL SÍNDROME DE RETT: INTERVENCIÓN EN UN CASO. International Journal of Developmental and Educational Psychology. 2006;3(1):375-86.

13. Lucía Inés Lahoza Estarriaga. El mutismo selectivo: diagnóstico, factores y pautas de intervención. Revista Arista Digital. 2013;(38):78-83

14. Miriam Rodríguez Menchón, Juan J. Saval Manera. Tratamiento conductual basado en el juego de una niña con mutismo selectivo. Revista de psicología clínica con niños y adolescentes. 2017;4(1):51-7.

15. Luckasson, Bortwick-Duffy, Buntinx, Coulter, Craig, Reeve, et al. Book Review. The psychological Record. 2003;53:327-9.

16. E. Fernández Álvarez. Estereotipias. Revista de neurología. 2003;36(1):54-6. 